\title{
Affine-evasive Sets Modulo a Prime
}

\author{
Divesh Aggarwal ${ }^{*}$
}

October 16, 2014

\begin{abstract}
In this work, we describe a simple and efficient construction of a large subset $S$ of $\mathbb{F}_{p}$, where $p$ is a prime, such that the set $A(S)$ for any non-identity affine map $A$ over $\mathbb{F}_{p}$ has small intersection with $S$.

Such sets, called affine-evasive sets, were defined and constructed in [ADL14] as the central step in the construction of non-malleable codes against affine tampering over $\mathbb{F}_{p}$, for a prime $p$. This was then used to obtain efficient non-malleable codes against split-state tampering.

Our result resolves one of the two main open questions in [ADL14]. It improves the rate of non-malleable codes against affine tampering over $\mathbb{F}_{p}$ from $\log \log p$ to a constant, and consequently the rate for non-malleable codes against split-state tampering for $n$-bit messages is improved from $n^{6} \log ^{7} n$ to $n^{6}$.
\end{abstract}

${ }^{*}$ Department of Computer Science, New York University. Email: divesha@cs.nyu.edu. 


\section{Introduction}

Non-malleable Codes (NMCs). NMCs were introduced in [DPW10] as a beautiful relaxation of error-correction and error-detection codes. Informally, given a tampering family $\mathcal{F}$, an NMC (Enc, Dec) against $\mathcal{F}$ encodes a given message $m$ into a codeword $c \leftarrow \operatorname{Enc}(m)$ in a way that, if the adversary modifies $m$ to $c^{\prime}=f(c)$ for some $f \in \mathcal{F}$, then the message $m^{\prime}=\operatorname{Dec}\left(c^{\prime}\right)$ is either the original message $m$, or a completely "unrelated value". As has been shown by the recent progress [DPW10, LL12, DKO13, ADL14, FMVW13, FMNV14, CG14a, CG14b] NMCs aim to handle a much larger class of tampering functions $\mathcal{F}$ than traditional error-correcting or errordetecting codes, at the expense of potentially allowing the attacker to replace a given message $x$ by an unrelated message $x^{\prime}$. NMCs are useful in situations where changing $x$ to an unrelated $x^{\prime}$ is not useful for the attacker (for example, when $x$ is the secret key for a signature scheme.)

Split-State Model. NMCs do not exist for the class of all functions $\mathcal{F}_{\text {all }}$. In particular, it does not include functions of the form $f(c):=\operatorname{Enc}(h(\operatorname{Dec}(c)))$, since $\operatorname{Dec}(f(\operatorname{Enc}(m)))=h(m)$ is clearly related to $m$. One of the largest and practically relevant tampering families for which we can construct NMCs is the so-called split-state tampering family where the codeword is split into two parts $c_{1} \| c_{2}$, and the adversary is only allowed to tamper with $c_{1}, c_{2}$ independently to get $f_{1}\left(c_{1}\right) \| f_{2}\left(c_{2}\right)$. A lot of the aforementioned results [LL12, DKO13, ADL14, CG14b, FMNV14] have studied NMCs against split-state tampering. [ADL14] gave the first (and the only one so far) information-theoretically secure construction in the split-state model from $n$-bit messages to $n^{7} \log ^{7} n$-bit codewords (i.e., code rate $n^{6} \log ^{7} n$ ). The security proof of this scheme relied on an amazing property of the inner-product function modulo a prime, that was proved using results from additive combinatorics.

Affine-evasive Sets and Our Result. One of the crucial steps in the construction of [ADL14] was the construction of NMC against affine tampering modulo $p$. This was achieved by constructing an affine-evasive set of size $p^{1 / \log \log p}$ modulo a prime $p$. It was asked as an open question whether there exists an affine-evasive set of size $p^{\Theta(1)}$, which will imply constant rate NMC against affine-tampering and rate $n^{6} \mathrm{NMC}$ against split-state tampering. ${ }^{1}$ We resolve this question in the affirmative by giving an affine-evasive set of size $\Theta\left(\frac{p^{1 / 4}}{\log p}\right)$.

\section{Explicit Construction}

For any set $S \subset \mathbb{Z}$, let $a S+b=\{a s+b \mid s \in S\}$. By $S \bmod p \subseteq \mathbb{F}_{p}$, we denote the set of values of $S$ modulo $p$.

We first define an affine-evasive set $S \subseteq \mathbb{F}_{p}$.

Definition 1 A non-empty set $S \subseteq \mathbb{F}_{p}$ is said to be $(\gamma, \nu)$-affine-evasive if $|S| \leq \gamma p$, and for any $(a, b) \in \mathbb{F}_{p}^{2} \backslash\{(1,0)\}$, we have

$$
|S \cap(a S+b \quad(\bmod p))| \leq \nu|S|
$$

\footnotetext{
${ }^{1}$ Under a plausible conjecture, this will imply constant rate NMC against split-state tampering. See Theorem 5 for more details.
} 
Now we give a construction of an affine-evasive set.

Let $Q:=\left\{q_{1}, \ldots, q_{t}\right\}$ be the set of all primes less than $\frac{1}{2} p^{1 / 4}$. Define $S \subset \mathbb{F}_{p}$ as follows:

$$
S:=\left\{\frac{1}{q_{i}}(\bmod p) \mid i \in[t]\right\} .
$$

Thus, $S$ has size $\Theta\left(\frac{p^{1 / 4}}{\log p}\right)$ by the prime number theorem.

Theorem 1 For any prime $p$, the set $S$ defined in Equation (1) is $\left(\frac{1}{2} p^{-3 / 4}, O\left(p^{-1 / 4} \cdot \log p\right)\right)$-affineevasive.

Proof. Clearly,

$$
|S|=t \leq \frac{1}{2} p^{1 / 4}=\frac{1}{2} p^{-3 / 4} \cdot p .
$$

Fix $a, b \in \mathbb{F}_{p}$, such that $(a, b) \neq(1,0)$. Now, we show that $|S \cap(a S+b(\bmod p))| \leq 3$. Assume, on the contrary, that there exist distinct $\alpha_{i} \in Q$ for $i \in\{0,1,2,3\}$ such that $1 / \alpha_{i}(\bmod p) \in S \cap(a S+b$ $(\bmod p))$. We have

$$
\frac{a}{\beta_{i}}+b=\frac{1}{\alpha_{i}}(\bmod p) \text { for } i=0,1,2,3,
$$

where $\beta_{i}, \alpha_{i} \in Q$ for $i \in\{0,1,2,3\}$, and $\alpha_{i} \neq \alpha_{j}$ for any $i \neq j$.

For any $i$, if $\beta_{i}=\alpha_{i}$, then $b \cdot \beta_{i}=1-a \bmod p$, which has at most one solution (since we assume $(a, b) \neq(1,0))$. Thus, without loss of generality, we assume that $\beta_{i} \neq \alpha_{i}$, for $i \in\{1,2,3\}$, and $\beta_{1}<\beta_{2}<\beta_{3}$.

From Equation (2), we have that

$$
\frac{\frac{a}{\beta_{1}}+b-\frac{a}{\beta_{2}}-b}{\frac{a}{\beta_{1}}+b-\frac{a}{\beta_{3}}-b}=\frac{\frac{1}{\alpha_{1}}-\frac{1}{\alpha_{2}}}{\frac{1}{\alpha_{1}}-\frac{1}{\alpha_{3}}} \quad(\bmod p),
$$

which on simplification implies

$$
\left(\alpha_{3}-\alpha_{1}\right)\left(\beta_{2}-\beta_{1}\right) \beta_{3} \alpha_{2}=\left(\alpha_{2}-\alpha_{1}\right)\left(\beta_{3}-\beta_{1}\right) \beta_{2} \alpha_{3} \quad(\bmod p) .
$$

Note that both the left-hand and right-hand side of the above equation takes values between $\frac{-p}{16}$ and $\frac{p}{16}$, and hence the equality holds in $\mathbb{Z}$ (and not just in $\mathbb{Z}_{p}$ ).

$$
\left(\alpha_{3}-\alpha_{1}\right)\left(\beta_{2}-\beta_{1}\right) \beta_{3} \alpha_{2}=\left(\alpha_{2}-\alpha_{1}\right)\left(\beta_{3}-\beta_{1}\right) \beta_{2} \alpha_{3} .
$$

By equation 3 , we have that $\beta_{3}$ divides $\left(\alpha_{2}-\alpha_{1}\right)\left(\beta_{3}-\beta_{1}\right) \beta_{2} \alpha_{3}$. Clearly, $\beta_{3}$ is relatively prime to $\alpha_{3}, \beta_{2}$, and $\beta_{3}-\beta_{1}$. Therefore, $\beta_{3}$ divides $\left(\alpha_{2}-\alpha_{1}\right)$. This implies

$$
\beta_{3} \leq\left|\alpha_{2}-\alpha_{1}\right| \text {. }
$$

Also, from equation 3 , we have that $\alpha_{2}$ divides $\left(\alpha_{2}-\alpha_{1}\right)\left(\beta_{3}-\beta_{1}\right) \beta_{2} \alpha_{3}$, which by similar reasoning implies $\alpha_{2}$ divides $\beta_{3}-\beta_{1}$. Thus, using that $\beta_{3}>\beta_{1}$,

$$
0<\alpha_{2} \leq \beta_{3}-\beta_{1}<\beta_{3} .
$$

Similarly, we can obtain $\alpha_{1}$ divides $\beta_{3}-\beta_{2}$, which implies

$$
0<\alpha_{1} \leq \beta_{3}-\beta_{2}<\beta_{3} .
$$

Equation (5) and (6) together imply that $\left|\alpha_{2}-\alpha_{1}\right|<\beta_{3}$, which contradicts Equation (4). 


\section{Affine-evasive function and Efficient NMCs}

Affine-evasive function. We recall here the definition of affine-evasive functions from [ADL14]. Affine-evasive functions immediately give efficient construction of NMCs against affine-tampering.

Definition $2 A$ surjective function $h: \mathbb{F}_{p} \mapsto \mathcal{M} \cup\{\perp\}$ is called $(\gamma, \delta$ )-affine-evasive if for any $a, b \in \mathbb{F}_{p}$ such that $a \neq 0$, and $(a, b) \neq(1,0)$, and for any $m \in \mathcal{M}$,

1. $\operatorname{Pr}_{U \leftarrow \mathbb{F}_{p}}(h(a U+b) \neq \perp) \leq \gamma$

2. $\operatorname{Pr}_{U \leftarrow \mathbb{F}_{p}}(h(a U+b) \neq \perp \mid h(U)=m) \leq \delta$

3. A uniformly random $X$ such that $h(X)=m$ is efficiently samplable.

We now mention a result that shows that we can construct an affine-evasive function from an affine-evasive set $S$.

Lemma 1 ([ADL14, Claim 5]) Let $S \subseteq \mathbb{F}_{p}$ be a $(\gamma, \nu)$-affine-evasive set with $\nu \cdot K \leq 1$, and $K$ divides $|S|{ }^{2} \quad$ Furthermore, let $S$ be ordered such that for any $i$, the $i$-th element is efficiently computable in $O(\log p)$. Then there exists a $(\gamma, \nu \cdot K)$-affine-evasive function $h: \mathbb{F}_{p} \mapsto \mathcal{M} \cup\{\perp\}$.

Note that the above result requires that for any $i$, the $i$-th element of $S$ is efficiently computable for some ordering of the set $S$. This is not possible for our construction since for our construction this would mean efficiently sampling the $i$-th largest prime. However, this requirement was made just to make sure that $h^{-1}$ is efficiently samplable. We circumvent this problem by giving a slightly modified definition of the affine-evasive function $h$ in the proof of Lemma 2. Before proving this, we state the following result that we will need.

Theorem 2 ([HB88]) For any $n \in \mathbb{N}$, and any $n^{\prime} \leq n$ such that $n^{\prime 12 / 7} \geq n$,

$$
\pi(n)-\pi\left(n-n^{\prime}\right)=\Theta\left(\frac{n^{\prime}}{\log n}\right),
$$

where $\pi(n)$ denote the number of primes less than $n$.

Lemma 2 Let $\mathcal{M}$ be a finite set such that $|\mathcal{M}| \geq 2$, and let $p \geq|\mathcal{M}|^{16}$ be a prime. There exists an efficiently computable $\left(p^{-3 / 4}, O\left(|\mathcal{M}| \log p \cdot p^{-1 / 4}\right)\right)$-affine-evasive function $h: \mathbb{F}_{p} \mapsto \mathcal{M} \cup\{\perp\}$.

Proof. Without loss of generality, let $\mathcal{M}=\{1, \ldots, K\}$, for some integer $K$. Let $S \subseteq \mathbb{F}_{p}$ be as defined in Section 2. Define $S_{1}, \ldots, S_{K}$ to be a partition of $S$ as follows.

$$
S_{i}:=\left\{s \in S \mid \frac{1}{s} \in\left[\frac{i-1}{2 K} p^{1 / 4}, \frac{i}{2 K} p^{1 / 4}\right)\right\} .
$$

Now let $n_{i}=\frac{p^{1 / 4} i}{2 K}$ and $n^{\prime}=\frac{p^{1 / 4}}{2 K}$. By the construction of $S,\left|S_{i}\right|=\pi\left(n_{i}\right)-\pi\left(n_{i}-n^{\prime}\right)$. We will bound $\left|S_{i}\right|$ for all $i \in[K]$ using Theorem 2. To do this, we need to verify that for all $i, n^{\prime 12 / 7} \geq n_{i}$. Since $n_{i}<n_{j}$ for all $i<j$, it is sufficient to show this for $i=K$, i.e., $n_{i}=\frac{p^{1 / 4}}{2}$.

$$
\frac{n^{12 / 7}}{n_{K}}=\frac{2 p^{3 / 7}}{(2 K)^{12 / 7} p^{1 / 4}}=\frac{p^{5 / 28}}{2^{5 / 7} \cdot K^{12 / 7}} \geq \frac{K^{5 \cdot 16 / 28}}{2^{5 / 7} \cdot K^{12 / 7}}=\frac{K^{8 / 7}}{2^{5 / 7}}>1,
$$

\footnotetext{
${ }^{2}$ The assumption $K$ divides $|S|$ is just for simplicity.
} 
where we used the fact that $p \geq K^{16}$, and $K \geq 2$. Also note that $n_{i}$ is upper bounded by $\frac{p^{1 / 4}}{2}$, and hence $\log n_{i}=O(\log p)$. Thus, using Theorem 2, we get that each $S_{i}$ has size at least $\Theta\left(\frac{p^{1 / 4}}{K \log p}\right)$.

Let $h: \mathbb{F}_{p} \mapsto \mathcal{M} \cup\{\perp\}$ be defined as follows:

$$
h(x)= \begin{cases}i & \text { if } x \in S_{i} \\ \perp & \text { otherwise }\end{cases}
$$

The statement $\operatorname{Pr}(h(a U+b) \neq \perp) \leq p^{-3 / 4}$ is obvious by the definition of $S$, and the observation that $a U+b$ is uniform in $\mathbb{F}_{p}$.

Also, for any $m \in \mathcal{M}$, and for any $(a, b) \neq(1,0)$, and $a \neq 0$,

$$
\begin{aligned}
\operatorname{Pr}(h(a U+b) \neq \perp \mid h(U)=m) & =\frac{\operatorname{Pr}\left(a U+b \in S \wedge U \in S_{m}\right)}{\operatorname{Pr}\left(U \in S_{m}\right)} \\
& \leq \frac{\operatorname{Pr}(a U+b \in S \wedge U \in S)}{\left|S_{m}\right| / p} \\
& =\frac{p}{\left|S_{m}\right|} \operatorname{Pr}\left(U \in S \cap\left(a^{-1} S-b a^{-1}\right) \quad(\bmod p)\right) \\
& =O\left(K \log p \cdot p^{-1 / 4}\right) .
\end{aligned}
$$

Also, sampling a uniformly random $X$ such that $h(X)=m$ is equivalent to sampling a uniformly random prime $q$ in the interval

$$
I:=\left[\frac{m-1}{2 K} p^{1 / 4}, \frac{m}{2 K} p^{1 / 4}\right)
$$

and computing $1 / q \bmod p$. Sampling $q$ can be done in time polynomial in $\log p$ by repeatedly sampling a random element in $I$ until we get a prime. Computing $1 / q \bmod p$ can be done efficiently using Extended Euclidean Algorithm.

Note that the proof of Lemma 2 is identical to the proof of Lemma 1, except the proof that a uniformly random $X$ such that $h(X)=m$ is efficiently samplable for any given $m$.

Efficient NMCs. We recall here the definition of non-malleable codes for completeness.

Definition 3 Let $\mathcal{F}$ be some family of tampering functions. For each $f \in \mathcal{F}$, and $m \in \mathcal{M}$, define the tampering-experiment

$$
\operatorname{Tamper}_{m}^{f}:=\left\{\begin{array}{c}
c \leftarrow \operatorname{Enc}(m), \tilde{c} \leftarrow f(c), \tilde{m}=\operatorname{Dec}(\tilde{c}) \\
\text { Output: } \tilde{m} .
\end{array}\right\}
$$

which is a random variable over the randomness of the encoding function Enc. We say that a coding scheme (Enc, Dec) is $\varepsilon$-non-malleable w.r.t. $\mathcal{F}$ if for each $f \in \mathcal{F}$, there exists a distribution (corresponding to the simulator) $D_{f}$ over $\mathcal{M} \cup\{\perp$, same* $\}$, such that, for all $m \in \mathcal{M}$, we have that the statistical distance between Tamper ${ }_{m}^{f}$ and

$$
\operatorname{Sim}_{m}^{f}:=\left\{\begin{array}{c}
\tilde{m} \leftarrow D_{f} \\
\text { Output: } m \text { if } \tilde{m}=\text { same*, and } \tilde{m}, \text { otherwise. }
\end{array}\right\}
$$

is at most $\varepsilon$. Additionally, $D_{f}$ should be efficiently samplable given oracle access to $f(\cdot)$. 
Using Lemma 2 and the construction of [ADL14], we get the following results.

Theorem 3 There exists an efficient coding scheme (Enc, Dec) encoding $k$-bit messages to $\Theta(k+$ $\left.\log \left(\frac{1}{\varepsilon}\right)\right)$ bit codewords that is $\varepsilon$-non malleable w.r.t. the family of affine tampering functions $\mathcal{F}_{\text {aff }}$.

Theorem 4 There exists an efficient coding scheme (Enc, Dec) encoding $k$-bit messages to $\Theta((k+$ $\left.\left.\log \left(\frac{1}{\varepsilon}\right)\right)^{7}\right)$ bit codewords that is $\varepsilon$-non malleable w.r.t. the family of split-state tampering functions $\mathcal{F}_{\text {split }}$.

Also, assuming the following conjecture from [ADL14], our result gives the first NMC with constant rate in the split-state model.

Conjecture 1 ([ADL14, Conjecture 2]) There exists absolute constants $c, c^{\prime}>0$ such that the following holds. For any finite field $\mathbb{F}_{p}$ of prime order, and any $n>c^{\prime}$, let $L, R \in \mathbb{F}_{p}^{n}$ be uniform, and fix $f, g: \mathbb{F}_{p}^{n} \rightarrow \mathbb{F}_{p}^{n}$. Let $\mathcal{D}$ be the family of convex combinations of $\left\{(U, a U+b): a, b \in \mathbb{F}_{p}\right\}$ where $U \in \mathbb{F}_{p}$ is uniform. Then there exists $D \in \mathcal{D}$ such that

$$
\Delta(\langle L, R\rangle,\langle f(L), g(R)\rangle ; D) \leq p^{-c n} .
$$

Theorem 5 Assuming Conjecture 1, there exists an efficient coding scheme (Enc,Dec) encoding $k$-bit messages to $\Theta\left(k+\log \left(\frac{1}{\varepsilon}\right)\right)$ that is $\varepsilon$-non malleable w.r.t. the family of split-state tampering functions $\mathcal{F}_{\text {split }}$.

\section{References}

[ADL14] D. Aggarwal, Y. Dodis, and S. Lovett. Non-malleable codes from additive combinatorics. In STOC, 2014. To appear.

[CG14a] M. Cheraghchi and V. Guruswami. Capacity of non-malleable codes. In Innovations in Theoretical Computer Science. ACM, 2014. To appear.

[CG14b] M. Cheraghchi and V. Guruswami. Non-malleable coding against bit-wise and split-state tampering. In Theory of Cryptography Conference - TCC. Springer, 2014. To appear.

[DKO13] Stefan Dziembowski, Tomasz Kazana, and Maciej Obremski. Non-malleable codes from twosource extractors. In Advances in Cryptology-CRYPTO 2013. Springer, 2013.

[DPW10] Stefan Dziembowski, Krzysztof Pietrzak, and Daniel Wichs. Non-malleable codes. In Andrew Chi-Chih Yao, editor, ICS, pages 434-452. Tsinghua University Press, 2010.

[FMNV14] S. Faust, P. Mukherjee, J. Nielsen, and D. Venturi. Continuous non-malleable codes. In Theory of Cryptography Conference - TCC. Springer, 2014. To appear.

[FMVW13] S. Faust, P. Mukherjee, D. Venturi, and D. Wichs. Efficient non-malleable codes and keyderivation for poly-size tampering circuits. IACR Cryptology ePrint Archive, 2013.

[HB88] D. R. Heath-Brown. The number of primes in a short interval. J. Reine Angew. Math., 389:2263, 1988.

[LL12] Feng-Hao Liu and Anna Lysyanskaya. Tamper and leakage resilience in the split-state model. In Advances in Cryptology-CRYPTO 2012, pages 517-532. Springer, 2012. 